\title{
UNIVERSAL PETTIS INTEGRABILITY PROPERTY
}

\author{
GUNNAR F. STEFÁNSSON
}

(Communicated by Dale Alspach)

\begin{abstract}
Functions into duals and pre-duals of weakly compactly generated spaces (WCG) are studied.

We show that a universally weakly measurable function $f$ into a dual of a WCG has the RS property. Also, for such a function, we sharpen the decomposition obtained by E. M. Bator (1988).

We show that bounded weakly measurable functions into pre-duals of WCG spaces are always Pettis integrable, universally weakly measurable, or not.
\end{abstract}

\section{INTRODUCTION}

A function $f$ defined on a compaci Hausdorff space $\Omega$ with values in a Banach space $X$ is said to be universally weakly measurable if, for every Radon measure $\mu$ on $\omega$ and each $x^{*}$ in $X^{*}$, the dual of $X$, the scalar-valued function $x^{*} f$ is $\mu$-measurable. In that case, $f$ is universally Pettis integrable if it is Pettis integrable against every Radon measure $\mu$ on $\Omega$.

The notion of the universal Pettis integrability property of Banach spaces (UPIP) was introduced in [5]. A Banach space $X$ has the UPIP if for every compact Hausdorff space $\Omega$, every universally weakly measurable function $f: \Omega \rightarrow X$ is universally Pettis integrable. In [5, Theorem 6] it was shown that duals of separable spaces have the UPIP. The proof reveals the following property of universally weakly measurable functions:

$\operatorname{RS}(D):\left\{\begin{array}{l}\text { For every Radon measure } \mu \text { on } \Omega \text { and every separable subspace } D \\ \text { of } X^{*} \text { the family } Z_{f}(D)^{*}=\left\{x^{*} f: x^{*} \in D,\left\|x^{*}\right\| \leq 1\right\} \text { is almost } \\ \text { weakly precompact in } L_{\infty}(\mu) .\end{array}\right.$

If $\operatorname{RS}(D)$ holds true for all subspaces $D$ of $X^{*}$, separable or not, then $f$ is said to have the RS property for $\mu$. Here RS stands for Riddle-Saab, terminology used by Talagrand in [7], where he asks if bounded universally weakly measurable functions always have the RS property.

A key step in the proof of [5, Theorem 6] is to show that universally weakly measurable functions into duals of separable spaces do have the RS property. In this paper we will show that a universally weakly measurable function into duals

Received by the editors July 14, 1993; contents presented at the 96th Summer Meeting of the AMS (Minneapolis Mathfest), Minneapolis, Minnesota, August 15-17, 1994.

1991 Mathematics Subject Classification. Primary 46G10, 28B05.

Key words and phrases. Universally weakly measurable, weak* integral, Pettis integral. 
of WCG spaces always has the RS property. Further, it is shown that, given any Radon measure $\mu$, any such function $f$ can be written as a sum of two universally weakly measurable functions, one being universally Pettis integrable and the other $\mu$-weak* equivalent to zero. It is still an open question whether a dual of a WCG space $X$ has the UPIP, but this decomposition shows that in order to determine which it is, it suffices to consider those functions that vanish almost everywhere against elements from $X$, that is, those for which $f x=0$ almost everywhere.

The UPIP is a restriction of the more general Pettis integrability property (PIP). A Banach space $X$ is said to have the PIP if for every finite and complete measure space $(\Omega, \Sigma, \lambda)$, every bounded weakly measurable function $f: \Omega \rightarrow$ $X$ is Pettis integrable. We will show that pre-duals of WCG spaces have the PIP.

\section{NOTATION AND TERMINOLOGY}

The dual of a Banach space $X$ will be denoted by $X^{*}$. Given a finite complete measure space $(\Omega, \Sigma, \mu)$, a bounded function $f: \Omega \rightarrow X$ is said to be weakly measurable if the scalar-valued function $x^{*} f$ is measurable for all $x^{*}$ in $X^{*}$. In that case, for each $E$ in $\Sigma$, the Dunford integral of $f$ over $E$ is the element of $x_{E}^{* *}$ defined by the equation $x_{E}^{* *}\left(x^{*}\right)=\int_{E} x^{*} f d \mu . f$ is said to be Pettis integrable if $x_{E}^{* *}$ is in $X$ for all $E$ in $\Sigma$.

If $X$ is a dual space, $X=Y^{*}, f$ is said to be weak* measurable if the scalar function $f y$ is measurable for all $y$ in $Y$. The weak ${ }^{*}$ integral of $f$ over $E$ is the element $y_{E}^{*}$ of $Y^{*}$ defined by the equation $y_{E}^{*}(y)=\int_{E} f y d \mu$.

A function $f: \Omega \rightarrow X\left(Y^{*}\right)$ is said to be $\mu$-weakly zero (resp. $\mu$-weak ${ }^{*}$ zero) if $x^{*} f$ (resp. $f y$ ) is zero $\mu$-a.e. Two functions $f$ and $g$ are called weakly equivalent (resp. weak ${ }^{*}$ equivalent) if their difference, $f-g$, is weakly zero (resp. weak * zero).

A function $f: \Omega \rightarrow X$ is said to be determined by a subspace $D$ of $X$ if for every $x^{*}$ in $X^{*},\left.x^{*}\right|_{D}=0$ implies that $x^{*} f=0 \mu$-a.e.

\section{THE RS PROPERTY}

We will need the following two lemmas, which we include for the sake of completeness; for proofs, see [6].

Lemma 1. Let $(\Omega, \Sigma, \lambda)$ be a finite complete measure space and $f: \Omega \rightarrow X^{*}$ be weak $^{*}$ integrable, and assume that the set $\{f x:\|x\| \leq 1\} \subset L_{1}(\lambda)$ is separable. Then $f$ is weak* equivalent to a weak* measurable function $g$ that takes its range in a weak* separable subspace of $X^{*}$.

The second lemma is based on a result of [3], where it is shown that a WCG space has a one-to-one continuous image of a reflexive space as dense subspace.

Lemma 2. Let $(\Omega, \Sigma, \lambda)$ be a finite complete measure space. If $X^{*}$ is a dual of WCG space $X$ and $f: \Omega \rightarrow X^{*}$ is weak ${ }^{*}$ integrable, then $\{f x:\|x\| \leq 1\} \subset$ $L_{1}(\lambda)$ is separable.

The above two lemmas together with the proof of [5, Theorem 6] allows us to prove the following:

Theorem 1. Let $X^{*}$ be a dual of a WCG space $X$, and let $f: \Omega \rightarrow X^{*}$ be a bounded universally weakly measurable function. For any Radon measure 
$\mu$ on $\Omega$, the family $\{f x:\|x\| \leq 1\}$ is almost weakly precompact in $L_{\infty}(\mu)$. Furthermore, $f$ can be written as a sum

$$
f=f_{1}+f_{2}
$$

where $f_{1}$ is universally Pettis integrable and $f_{2}$ is $\mu$-weak* equivalent to zero.

Proof. Let $\mu$ be any Radon measure on $\Omega$. By the above two lemmas, $f$ is weak * equivalent to a weak * measurable function $g: \Omega \rightarrow X^{*}$, that takes its range in a weak ${ }^{*}$ separable subspace of $X^{*}$. By a theorem of Amir and Lindenstrauss [1], we can write $X$ as a direct sum $X=X_{1} \oplus X_{2}$, where $X_{2}$ is separable and $g$ takes its range in $X_{2}^{*}$.

Since $X_{2}$ is separable, there exists a set $E \in \Sigma$ with $\mu(E)=0$ such that

$$
f x_{2}(\omega)=g x_{2}(\omega), \quad x_{2} \in X_{2}, \omega \in \Omega \backslash E .
$$

Consequently, for any $x_{2}^{* *} \in X_{2}^{* *}$

$$
x_{2}^{* *} f=x_{2}^{* *} g \text { a.e. }
$$

and hence, $g$ is weakly measurable.

Write $f$ as a sum,

$$
f=f_{1}+f_{2},
$$

where $f_{i}$ takes its range in $X_{i}^{*}, i=1,2$. It is clear that both $f_{1}$ and $f_{2}$ are universally weakly measurable. Since $f_{2}$ is valued in a dual of a separable space, it is universally Pettis integrable [5], and since for any $x$ in $X$,

$$
\begin{aligned}
f x & =g x \quad \mu \text {-a.e. } \\
& =f_{2} x,
\end{aligned}
$$

it follows that $f_{1}$ is weak ${ }^{*}$ equivalent to zero.

The two sets $Z_{f}\left(B_{X}\right)=\{f x: x \in X,\|x\| \leq 1\}$ and $Z_{g}\left(B_{X}\right)=\left\{g x_{2}: x_{2} \in\right.$ $\left.X_{2},\left\|x_{2}\right\| \leq 1\right\}$ are equal as subsets of $L_{\infty}(\mu)$, and since $g$ is valued in the dual of a separable space, the set $Z_{g}\left(B_{X}\right)$, and thus $Z_{f}\left(B_{X}\right)$, is almost weakly precompact in $L_{\infty}(\mu)$.

Since the measure $\mu$ was an arbitrary Radon measure on $\Omega$, the proof is complete.

Remark 1. In [2] Bator shows that universally weakly measurable functions are Pettis decomposable; that is, for any Radon measure $\mu$, any universally weakly measurable function $f$ can be written as a sum $f=f_{1}+f_{2}$ where $f_{2}$ is $\mu$-Pettis integrable and $f_{1}$ is $\mu$-weak * equivalent to zero. This decomposition can be done regardless of the Banach space in which the functions take their range. However, the functions $f_{1}$ and $f_{2}$ need not be weakly universally measurable, nor need either function be integrable with respect to other Radon measures.

Corollary 1. Let $X^{*}$ be a dual of a WCG space. The following are equivalent.

(1) $X^{*}$ has the UPIP.

(2) Given any compact Hausdorff space $\Omega$ and any Radon measure $\mu$ on $\Omega$, for any universally weakly measurable function $f: \Omega \rightarrow X^{*}$, the set $\boldsymbol{Z}_{f}\left(\boldsymbol{B}_{\boldsymbol{X}}\right)$ is dense in $\boldsymbol{Z}_{f}\left(\boldsymbol{B}_{\left.X^{* *}\right)}\right.$.

(3) Given any compact Hausdorff space $\Omega$ and any Radon measure $\mu$ on $\Omega$, every universally weakly measurable function $f: \Omega \rightarrow X^{*}$ which is $\mu$-weak* zero is weakly zero. 
Proof. (1) $\Rightarrow(2) \Rightarrow(3)$ is clear. (3) $\Rightarrow$ (1) follows from Theorem 1 .

Remark 2. If $X$ is not WCG, it is possible to construct a universally weakly measurable function $f$ valued in $X^{*}$ such that

$$
\{f x: x \in X\}=\left\{x^{* *} f: x^{* *} \in X^{* *}\right\}
$$

but $f$ fails to be universally Pettis integrable. Indeed, Phillips [4] has given an example of a weakly Borel, and therefore universally weakly measurable function $f:[0,1] \rightarrow l_{\infty}[0,1]$ which is weak* zero, but for any $x^{*}$ in $l_{\infty}[0,1]^{*} \backslash$ $l_{1}[0,1]$,

$$
x^{*} f=x^{*}\left(\chi_{[0,1]}\right) \quad(\text { constant }) \text { Lebesgue-a.e. }
$$

If we now define $\tilde{f}$ by the equation $\tilde{f}=f+\chi_{[0,1]}$, then $\tilde{f}$ is universally weakly measurable, not universally Pettis integrable because $f$ is not. However, for any $x^{*}$ in $l_{\infty}[0,1]^{*} \backslash l_{1}[0,1]$,

$$
x^{*} \tilde{f}=2 x^{*}\left(\chi_{[0,1]}\right)=\left\{2 x^{*}\left(\chi_{[0,1]}\right)\right\} \cdot \alpha(\tilde{f}),
$$

where $\alpha$ is any element of $l_{1}[0,1]$ satisfying $\alpha\left(\chi_{[0,1]}\right)=1$.

Theorem 2. If $X$ has a WCG dual, then $X$ has the PIP.

Proof. Let $(\Omega, \Sigma, \lambda)$ be any finite complete measure space, and let $f: \Omega \rightarrow$ $X$ be bounded and weakly measurable. We want to show that $f$ is Pettis integrable. To that end, we show that $f$ is determined by a separable subspace of $X$, and the result follows from [6].

View $f$ as a function into $X^{* *}$. By Lemmas 1 and $2, f$ is weak* equivalent to a weak* measurable function $g: \Omega \rightarrow X^{* *}$ that takes its range in a weak* separable subspace $Y$ of $X^{* *}$. This means that whenever $\left.x^{*}\right|_{Y}=0$ it follows that $g x^{*}(\omega)=0$ for almost all $\omega$, and hence $x^{*} f=0$ almost everywhere.

Let $\left\{y_{n}: n \in \mathbb{N},\left\|y_{n}\right\| \leq 1\right\}$ be a weak* dense subset of the unit ball of $Y$; and for each $n$, find a bounded sequence $\left(x_{n k}\right)$ in $X$ such that

$$
x_{n k} \rightarrow y_{n}
$$

weak*. If $D$ is the span of $\left\{x_{n k}: n, k \in \mathbb{N}\right\}$, then $D$ is a separable subspace of $X$ and $f$ is determined by $D$. Indeed, if $\left.x^{*}\right|_{D}=0$, then $x^{*}\left(x_{n k}\right)=0$ for all $n$ and $k$ and, hence, $y_{n}\left(x^{*}\right)=0$ for all $n$. Consequently, $x^{*} \mid Y=0$ and thus $x^{*} f=0$ almost everywhere.

Remark 3. Since $X^{*}$ is WCG, it has the Radon-Nikodym property (RNP). In particular, $X$ fails to contain a copy of $l_{1}$. It follows that not only does $X$ have the PIP, but also the indefinite integral of every Pettis integrable function $f: \Omega \rightarrow X$ has a relatively compact range. This holds true whether the function is bounded or not.

This is in some sense the closest functions into preduals of WCG spaces have come to imitating strongly measurable functions. In general, these functions are not weakly equivalent to strongly measurable functions. For example, all duals of the James Tree space have the PIP and the weak RNP. However, only the even duals (the WCG ones) have the RNP. 


\section{REFERENCES}

1. D. Amir and J. Lindenstrauss, The structure of weakly compact sets in Banach spaces, Ann. of Math. (2) 80 (1968), 34-46.

2. E. M. Bator, Pettis decomposition for universally scalarly measurable functions, Proc. Amer. Math. Soc. 104 (1988), 795-800.

3. W. J. Davis, T. Figiel, W. B. Johnson, and A. Pelczynski, Factoring weakly compact operators, J. Funct. Anal. 17 (1974), 311-327.

4. R. Phillips, Integrability in a convex linear topological space, Trans. Amer. Math. Soc. 47 (1940), 114-145.

5. L. H. Riddle, E. Saab, and J. J. Uhl, Sets with the weak Radon-Nikodym property in dual Banach spaces, Indiana Univ. Math. J. 32 (1978), 845-886.

6. G. F. Stefánsson, Pettis integrability, Trans. Amer. Math. Soc. 330 (1992), 401-418.

7. M. Talagrand, Pettis integral and measure theory, Mem. Amer. Math. Soc., no. 307, Amer. Math. Soc., Providence, RI, 1984.

Pennsylvania State University, Altoona Campus, Altoona, Pennsylvania 16601-3794

E-mail address: gf s@math.psu.edu 